\title{
Identification of candidate genes associated with cell wall digestibility and eQTL (expression quantitative trait loci) analysis in a Flint $\times$ Flint maize recombinant inbred line population Chun Shi ${ }^{1}$, Anna Uzarowska1, Milena Ouzunova ${ }^{2}$, Matthias Landbeck ${ }^{2}$, Gerhard Wenzel ${ }^{1}$ and Thomas Lübberstedt*3
}

Address: ${ }^{1}$ Chair of Plant Breeding, Technical University of Munich, Am Hochanger 2, 85350 Freising, Germany, ${ }^{2}$ KWS Saat AG, Grimsehlstrasse 31, 37555 Einbeck, Germany and ${ }^{3}$ Department of Genetics and Biotechnology, Research Centre Flakkebjerg, 4200 Slagelse, Denmark

Email: Chun Shi - marcuss@botany.utoronto.ca; Anna Uzarowska - anna.uzarowska@wzw.tum.de; Milena Ouzunova - m.ouzunova@kws.de; Matthias Landbeck - m.landbeck@KWS.de; Gerhard Wenzel - gwenzel@wzw.tum.de; Thomas Lübberstedt* - Thomas.Luebberstedt@agrsci.dk

* Corresponding author

Published: 18 January 2007

BMC Genomics 2007, 8:22 doi:10.1/86/147|-2164-8-22

This article is available from: http://www.biomedcentral.com//47I-2/64/8/22

(C) 2007 Shi et al; licensee BioMed Central Ltd.

This is an Open Access article distributed under the terms of the Creative Commons Attribution License (http://creativecommons.org/licenses/by/2.0), which permits unrestricted use, distribution, and reproduction in any medium, provided the original work is properly cited.

\begin{abstract}
Background: Cell-wall digestibility is the major target for improving the feeding value of forage maize. An understanding of the molecular basis for cell-wall digestibility is crucial towards breeding of highly digestible maize.

Results: 865 candidate ESTs for cell-wall digestibility were selected according to the analysis of expression profiles in I) three sets of brown-midrib isogenic lines in the genetic background of inbreds I332 (I332 and I332 bm3), 536I (536I and $536 \mathrm{l} \mathrm{bm} 3$ ), and F2 (F2, F2 bm , F2 bm2, and F2 bm3), 2) the contrasting extreme lines of FD (Flint $\times$ Dent, ASO8 $\times$ AS 06), DDI (Dent $\times$ Dent, ASII $\times$ AS09), and DD2 (Dent $\times$ Dent, AS29 $\times$ AS30) mapping populations, and 3) two contrasting isogenic inbreds, AS20 and AS2I. Out of those, 439 ESTs were assembled on our "Forage Quality Array", a small microarray specific for cell wall digestibility related experiments. Transcript profiles of 40 lines of a Flint $\times$ Flint population were monitored using the Forage Quality Array, which were contrasting for cell wall digestibility. Using $t$ tests $(p<0.01)$, the expression patterns of I02 ESTs were significantly different between high and low quality groups. Using interval mapping, eQTL (LOD $\geq 2.4$ ) were detected for $20 \%$ (89 of 439) of the spotted ESTs. On average, these eQTL explained $39 \%$ of the transcription variation of the corresponding ESTs. Only $26 \%$ ( 23 of 89 ) ESTs detected a single eQTL. eQTL hotspots, containing greater than $5 \%$ of the total number of eQTL, were located in chromosomal bins 1.07 , I.I2, 3.05, 8.03, and 9.04, respectively. Bin 3.05 was co-localized with a cell-wall digestibility related QTL cluster.
\end{abstract}

Conclusion: 102 candidate genes for cell-wall digestibility were validated by genetical genomics approach. Although the cDNA array highlights gene types (the tested gene and any close family members), trans-acting factors or metabolic bottlenecks seem to play the major role in controlling heritable variation of gene expression related to cell-wall digestibility, since no in silico mapped ESTs were in the same location as their own eQTL. Transcriptional variation was generally found to be oligogenic rather than monogenic inherited due to only $26 \%$ ESTs detected a single eQTL in the present study. One eQTL hotspot was co-localized with cell wall digestibility related QTL cluster on bins 3.05 , implying that in this case the gene(s) underlying QTL and eQTL are identical. As the field of genetical genomics develops, it is expected to significantly improve our knowledge about complex traits, such as cell wall degradability. Comprehensive knowledge of the lignin pathway and cell wall biogenesis will allow plant breeders to choose the best genomic targets controlling these characters, for improving forage digestibility through genetic engineering or marker-assisted selection. 


\section{Background}

Lignin content is well known as a major factor affecting forage quality in maize. However, correlations between lignin content and forage quality can be variable according to the genetic background [1]. Moreover, breeding for a higher digestibility of maize involves also other, so far unknown mechanisms [1]. Correlations between maize whole plant digestibility and cell-wall (or stover) digestibility ranged from 0.60 to 0.96 , with average values close to 0.80 , whereas correlations between whole-plant digestibility and grain or ear content were close to 0.4 [2]. Therefore, cell-wall digestibility is the major target for improving the feeding value of forage maize. An understanding of the molecular basis for cell-wall digestibility is crucial towards breeding of highly digestible maize.

An important first step to elucidate the mechanisms underlying cell-wall degradability is to identify causative genome regions. Five major QTL clusters involved in cellwall digestibility, located on chromosomal bins [3] 1.03, $3.05 / 06,6.06,8.05$, and 9.02, were identified by several QTL analyses, but the genes underlying these QTL are not yet known [1]. A second, more recent approach is transcriptome analysis to simultaneously measure the expression of thousands of genes. In comparison with normal maize genotypes, brown-midrib $(\mathrm{bm})$ mutants show a significantly reduced lignin content, altered lignin composition, and/or a significantly higher cell-wall digestibility [4]. Molecular mechanisms underlying cell wall digestibility in maize have been studied in three sets of maize brown-midrib isogenic lines in the genetic background of inbreds 1332 (1332 and 1332 bm3), 5361 (5361 and $5361 \mathrm{bm} 3$ ), and F2 (F2, F2 bm1, F2 bm2, and F2 bm3) [5]. 53 ESTs were differentially expressed in all three isogenic bm 3 comparisons, whereas 32 ESTs were consistently differentially expressed in different $b m$ isogenic lines in F2 background. Moreover, gene expression studies can be conducted on phenotypically extreme lines from mapping populations. Replicate pools of extreme lines can be profiled independently, so that differences in gene expression will be specific to the differing pools. This strategy was recently used to identify candidate genes for drought response QTL in rice [6]. Thus, by synthesis of expression profiling data from $\mathrm{bm}$ mutants and extreme lines of a mapping population segregating for cell wall digestibility, it should be possible to identify candidate genes related to cell-wall degradability, and to construct a microarray enriched for candidate genes underlying cell wall digestibility. Transcriptome analysis using such microarrays would provide a fingerprint of cell-wall metabolism in maize.

Genetic and gene expression approaches have been joined in the concept of "genetical genomics" [7], which aims to detect eQTL (expression quantitative trait loci) control- ling gene expression differences. Often, eQTL map to the genetic position of the respective gene itself, indicating that cis changes (within the gene) are responsible for the different levels of expression. In contrast, genes revealing (trans) eQTL at positions different from the genetic position of the respective gene are thought to be regulated by, e.g., trans-acting factors controlling their expression levels [8]. Detection of the master regulators, affecting expression levels of groups of genes, is a major feature of eQTL studies [9]. In plants, this strategy has been successfully applied to 76 maize lines in a F3 population [10] and 91 poplar lines in a backcross population [11], respectively. Altogether, combining expression profiling with genetic analysis could enrich our understanding of regulatory networks underlying cell-wall digestibility and assist plant breeders to choose the most relevant genomic targets for improvement of silage maize digestibility.

The objectives of our study were to 1) select candidate ESTs for cell-wall digestibility to establish a "Forage Quality Array", 2) identify ESTs differentially expressed between low and high digestible lines in a Flint $\times$ Flint mapping population, and 3) detect eQTL using the "genetical genomics" approach.

\section{Results \\ Selection of candidate ESTs in association with cell wall digestibility}

In order to identify genes in association with cell-wall digestibility in maize, three sources of genetic material were used, including 1) three sets of brown-midrib isogenic lines in the genetic background of inbreds 1332 (1332 and $1332 \mathrm{bm} 3$ ), 5361 (5361 and $5361 \mathrm{bm} 3$ ), and F2 (F2, F2 bm1, F2 bm2, and F2 bm3) [5], 2) the contrasting extreme lines of FD, DD1, and DD2 DH mapping populations, and 3) two isogenic inbreds, AS20 and AS21, significantly differing in cell wall digestibility. Two complementary approaches, SSH (suppression subtractive hybridization) and microarray-based expression profiling, were used to isolate and identify candidate genes in all comparisons. About $70 \%$ of the ESTs isolated by $\mathrm{SSH}$ were absent on the unigene microarray [5].

The number of at least two-fold induced ESTs in stems from SSH and microarray analyses, respectively, were 246 and 1417 in FD-pop (Figure 1), 122 and 317 in DD1-pop, 71 and 1805 in DD2-pop, as well as 225 and 630 in AS20 vs. AS21 (Data not shown). In FD-pop, 34 ESTs were jointly differentially expressed both in SSH and microarray experiments, as well as 8, 33, and 31 ESTs in DD1-pop, DD2-pop, and AS20 vs. AS21, respectively. The numbers of genes simultaneously differentially expressed in pairs of mapping populations were 145 (between FD-pop and DD1-pop), 374 (between FD-pop and DD2-pop), and 135 (between DD1-pop and DD2-pop), respectively. 58 
ESTs were consistently differentially expressed across the three mapping populations.

In total, 5460 distinct ESTs differentially expressed in one or multi- comparisons were identified. In order to verify the most interesting of those genes at low costs, a small microarray specific for cell wall digestibility (Forage Qual- ity Array) was designed. The first selection criterion (Figure 2) covered jointly differentially expressed ESTs both in SSH and microarray experiments for each comparison (287 ESTs). Secondly, 460 ESTs were selected with a more than five-fold change in respective comparisons. Thirdly, 91 EST homologues of lignin-related genes, PAL, 4CL, $\mathrm{C} 3 \mathrm{H}, \mathrm{CCOAOMT}$, CCR, COMT, and CAD, were spotted.

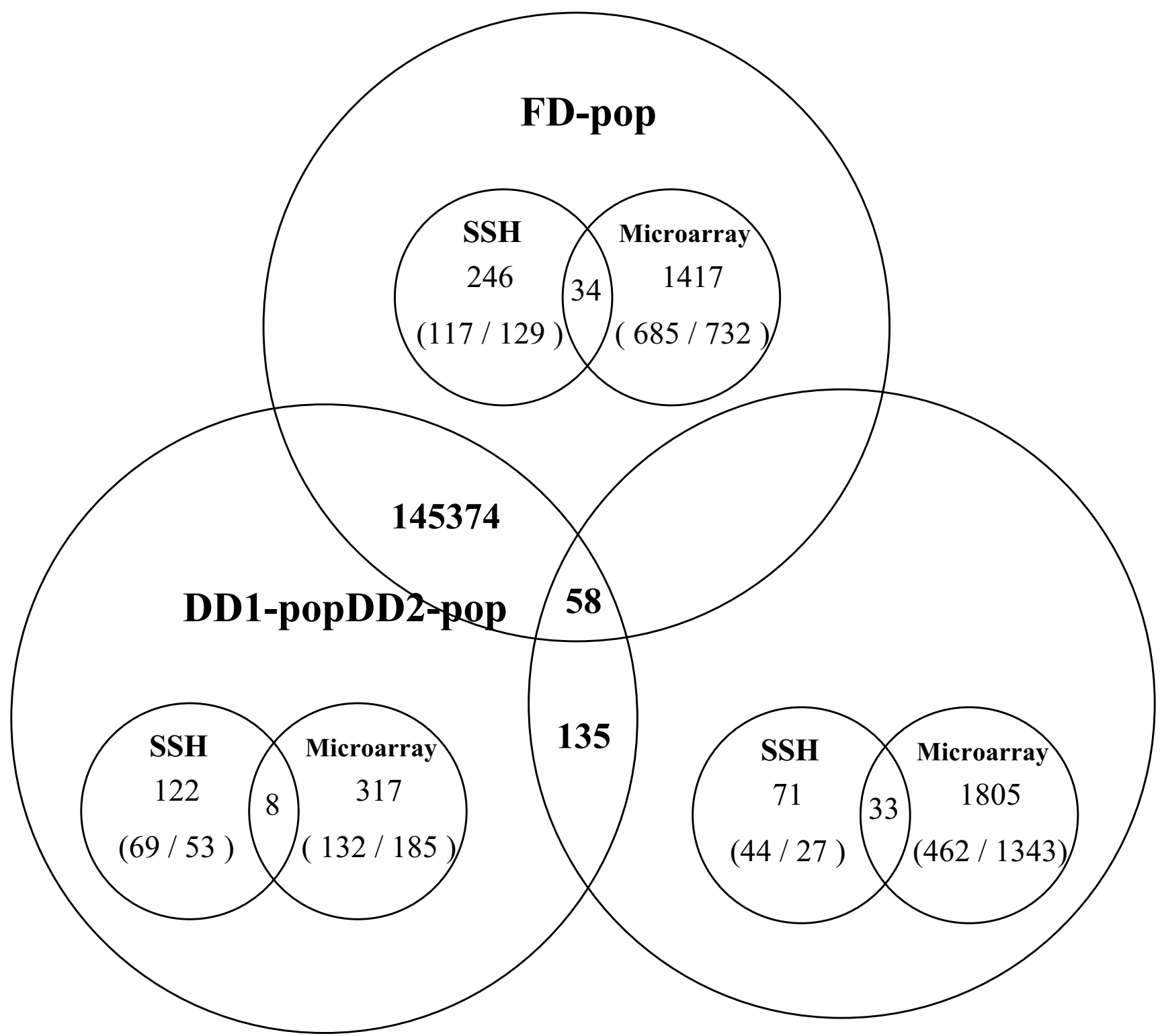

Figure I

Venn diagram for the summary of differentially expressed ESTs identified from FD (Flint $\times$ Dent, AS08 $\times$ AS 06), DDI (Dent $\times$ Dent, AS I I x AS09), and DD2 (Dent $\times$ Dent, AS29 × AS30) mapping populations, respectively. Total numbers of genes differentially expressed are indicated in respective circles. In parentheses, the first number indicates up-, the last number down-regulated genes. The number of genes simultaneously up- or down-regulated is given in intersections between circles. 
Subsequently, 115 ESTs in silico mapping to chromosomes 1,4 , and 5 were chosen, since $b m 1, b m 2$, and $b m 3$ map to these chromosomes. Finally, 53, 32, and 58 consistently differentially expressed ESTs in (i) all three bm3isogenic comparisons (1332 vs. 1332 bm 3, 5361 vs. 5361 bm3, and F2 vs. F2 bm3), (ii) all three isogenic comparisons in F2 background (F2 vs. F2 bm1, F2 vs. F2 bm2, and F2 vs. F2 bm3), and (iii) three mapping populations, respectively, were selected. According to the above mentioned criteria, 865 different candidate ESTs were identified towards production of the Forage Quality Array.

Out of 865 candidate ESTs, 151 were not available for ordering, and for 275 ESTs it was not possible to obtain high-quality PCR products due to poor bacteria recovery, unspecific PCR amplification, etc. Finally, 439 ESTs were included on the Forage Quality Array. GO vocabularies were assigned according to the TIGR Maize Gene Index [12]. $66 \%$ of these ESTs (288 of 439) could not be functionally classified. Among 151 classified ESTs, the largest category was "catalytic activity" (42\%) (Figure 3). Further ranking of classification categories was "binding" (28\%), "structural molecule activity" (12\%), and "transporter activity" (8\%).

Using hierarchical cluster analysis based on the EST tree, distinct expression patterns between high and low quality groups were found for most ESTs included on the Forage Quality Array (Figure 4). Using $t$-tests with a $p$ value cutoff of $0.01,102$ ESTs were significantly different between the two groups of high and low quality lines, including 39 down- and 63 up-regulated ESTs in the high quality group, respectively (Table 1). Out of 39 down-regulated ESTs in the high quality group, 1, 8, and 5 ESTs were clas-

a) Common ESTs between SSH and microarray for each comparison

\begin{tabular}{|c|c|c|c|c|c|c|c|c|c|}
\hline Comparison & $\begin{array}{c}1332 / \\
1332 \text { bm } 3\end{array}$ & $\begin{array}{c}5361 / \\
5361 \text { bm3 }\end{array}$ & $\begin{array}{c}\text { F2 / } \\
\text { F2 bm1 }\end{array}$ & $\begin{array}{c}\mathrm{F} 2 / \\
\mathrm{F} 2 \mathrm{bm} 2\end{array}$ & $\begin{array}{c}\text { F2 / } \\
\text { F2 bm3 }\end{array}$ & FD-pop & DD1-pop & DD2-pop & $\begin{array}{c}\text { AS 20 / } \\
\text { AS 21 }\end{array}$ \\
\hline EST number & 49 & 102 & 42 & 43 & 25 & 34 & 8 & 33 & 31 \\
\hline
\end{tabular}

b) Differentially expressed ESTs with at least five-fold change for each comparison

\begin{tabular}{lccccccccc}
\hline Comparison & $\mathbf{1 3 3 2} /$ & $\mathbf{5 3 6 1} /$ & $\mathbf{F 2} /$ & $\mathbf{F 2} /$ & $\mathbf{F 2} /$ & FD-pop & DD1-pop & DD2-pop & $\begin{array}{c}\text { AS 20/ } \\
\text { AS 21 }\end{array}$ \\
\hline EST number & 18 & 90 & 25 & 30 & 83 & 42 & 78 & 42 & 80 \\
\hline
\end{tabular}

c) EST homologs of the genes involved in lignin biosynthesis pathway

\begin{tabular}{lccccccc}
\hline Ligningene & PAL & 4CL & C3H & CCOAOMT & CCR & COMT & CAD \\
\hline EST number & 10 & 22 & 9 & 5 & 19 & 18 & 17 \\
\hline
\end{tabular}

d) In silico mapped ESTs located in chromosome 5, 1, and 4

\begin{tabular}{lccc}
\hline Chromosome & $\mathbf{1}$ & $\mathbf{4}$ & $\mathbf{5}$ \\
\hline EST number & 95 & 47 & 26 \\
\hline
\end{tabular}

e) Commonly differentially expressed ESTs in all three bm3-isogenic comparisons (1332/1332 bm3, 5361/5361 bm3, and F2/F2 bm3)

f) Commonly differentially expressed ESTs in all three isogenic comparisons $(b m 1, b m 2, b m 3)$ in $\mathrm{F} 2$ background

g) Commonly differentially expressed ESTs in FD, DD1, and DD2 mapping populations
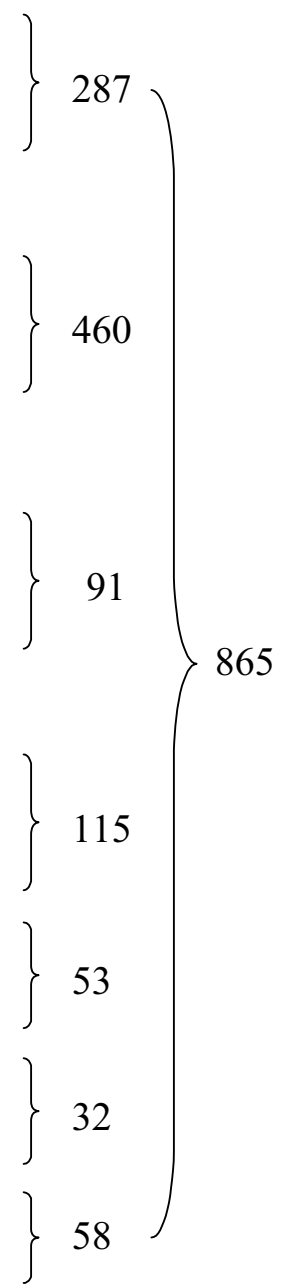

Figure 2

Selection of 865 candidate ESTs for cell wall digestibility based on seven selection criteria. 


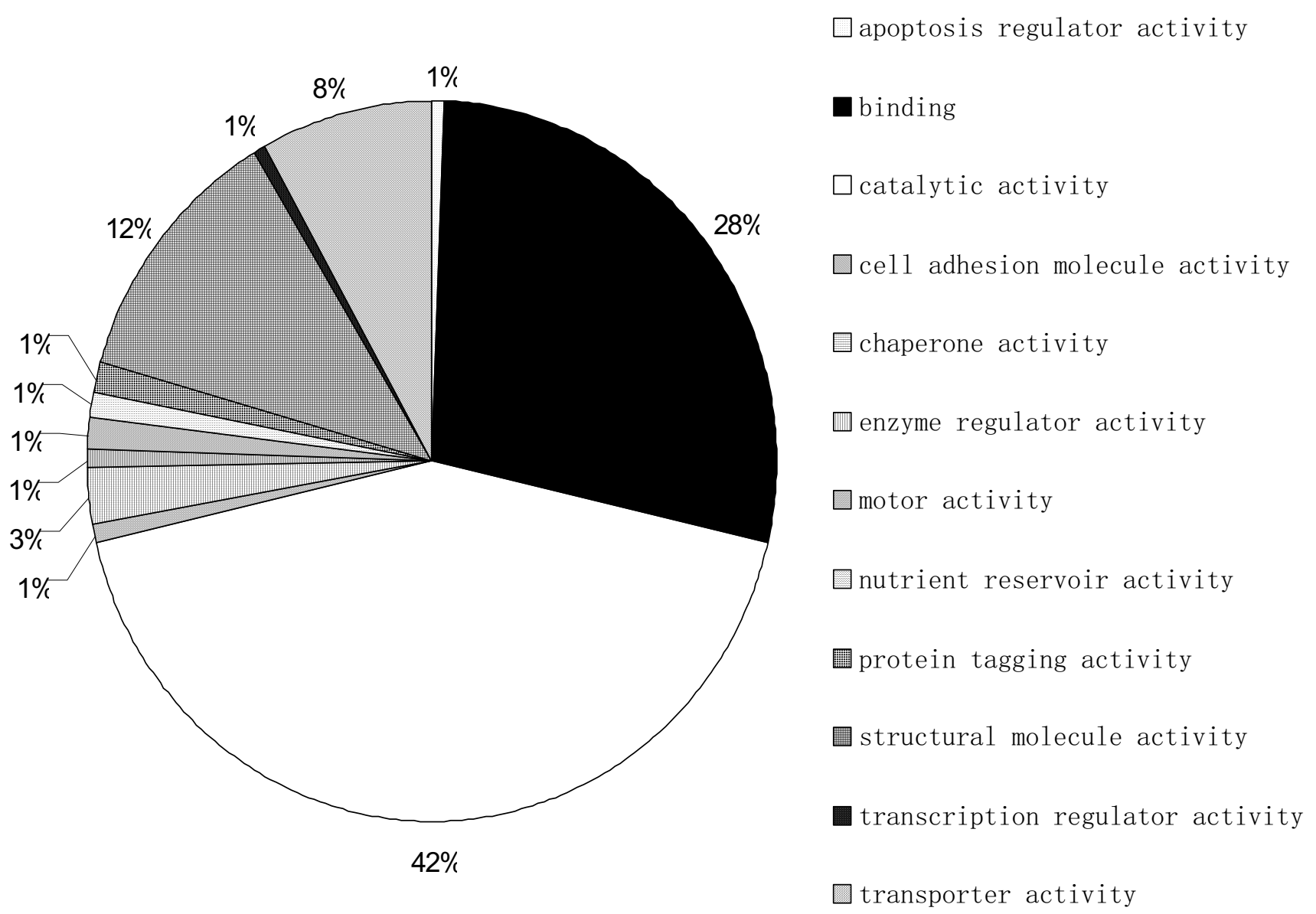

Figure 3

Assignment of the 439 ESTs spotted on the "Forage quality array" to functional classes by GO vocabularies. Each EST was assigned with GO vocabularies according to the TIGR Maize Gene Index [12]. Unclassified ESTs (66\%) were not taken into account.

sified into "chaperone activity", "catalytic activity", and "binding", respectively, whereas 1, 11, 2, 1, and 4 ESTs of up-regulated ESTs were classified into "motor activity", "catalytic activity", "structural molecular activity", "transporter activity" and "binding", respectively. According to the current annotation in the TIGR Maize Gene Index [12], four ESTs encoded enzymes involved in lignin biosynthesis, CD970581 (Cinnamyl-alcohol dehydrogenase), CD973094 (Phenylalanine ammonia-lyase), AW120445 (Class III peroxidase 67 precursor) and CF243853 (Caffeoyl CoA 3-O-methyltransferase). In addition, AI622068 (Cellulose synthase-2) is a homologue encoding a cellulose biosynthesis related enzyme, and BM333894 (YABBY-like transcription factor) and BU093700 (transcription factor and jumonji family protein) are putative transcription factors.

\section{Genetical genomics analysis}

40 extreme lines in the FF population with respect to dNDF were selected to represent two contrasting high and low quality groups (Table 2). The dNDF mean and SD of the low quality group was $52.34 \%$ and 2.12 , and $62.30 \%$ and 1.32 in the high quality group.

Using interval mapping, eQTL ( $L O D \geq 2.4$ ) were detected for $20 \%$ ( 89 of 439 ) of the tested ESTs, with a maximum LOD score of 5.2. On average, these eQTL explained 39\% of the genetic variation of the corresponding EST expression profiles, ranging from $24.1 \%$ to $91.4 \%$. The number of eQTL per EST varied from 1 to 8 with a mean of 3 (Figure $5 \mathrm{a}$ ). For $63 \%$ ( 56 of 89 ) ESTs, one to three eQTL were found. For four ESTs more than $6 \mathrm{eQTL}$ were detected. $39 \%$ (35 of 89) ESTs with eQTLs were significant for high and low pools as well. 


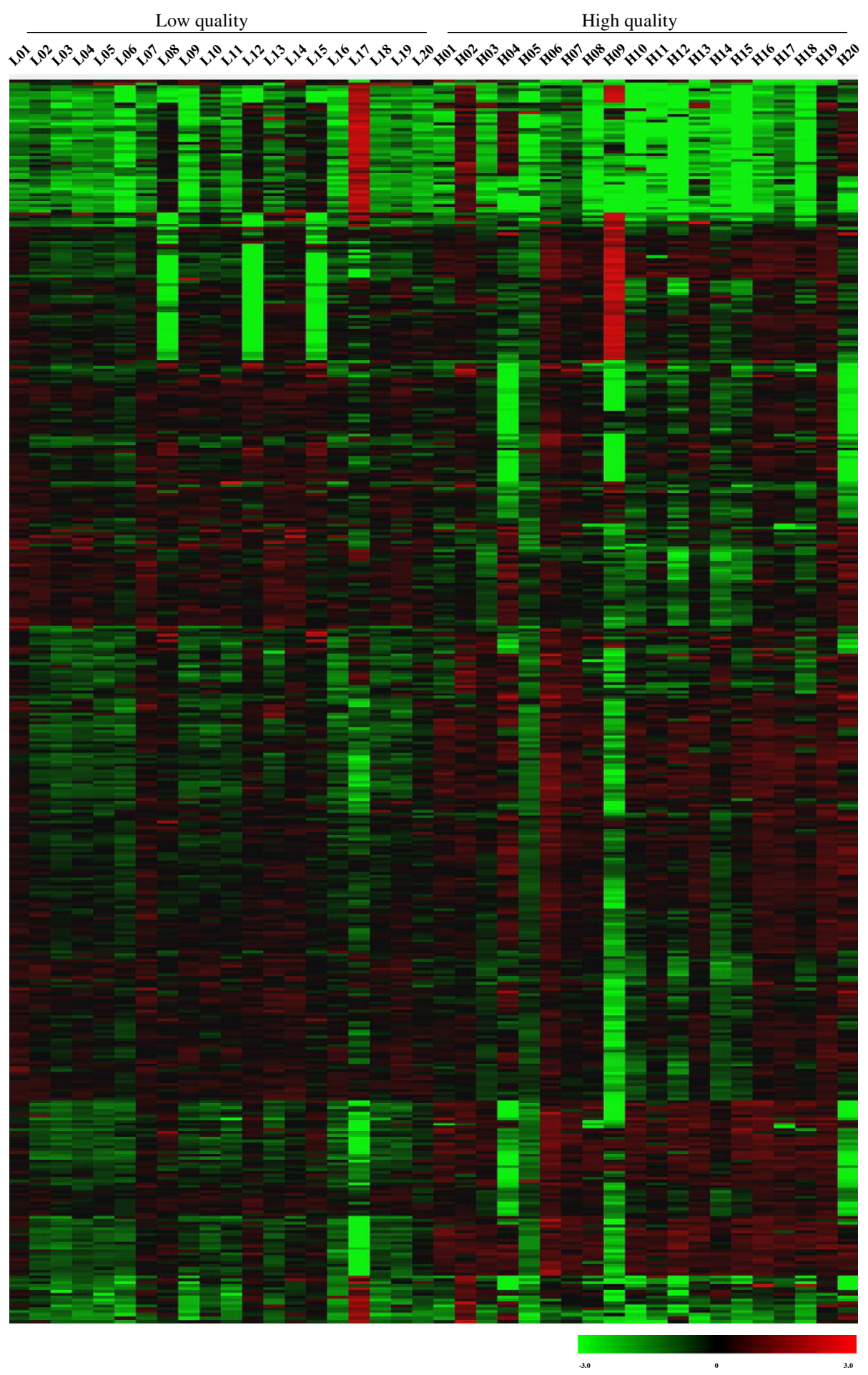

\section{Figure 4}

The expression profiling of 439 ESTs in $2 \times 20$ recombinant inbred lines contrasting for dNDF. The color saturation reflects the magnitude of the log 2 expression ratio (Cy5/Cy3) for each transcript. Each row represents an EST. Red and green colors mark up-regulated and down-regulated ESTs in individual line, respectively. The color log2 scale is provided at the bottom of this figure. 
Table I: Differentially expressed ( $p<0.01)$ ESTs $(102)$ between low and high digestible (dNDF) recombinant inbred lines.

\begin{tabular}{|c|c|c|c|c|c|c|}
\hline \multirow[t]{3}{*}{$\mathbf{G A}^{\mathrm{a}}$} & \multirow[t]{3}{*}{ Tentative annotation } & \multirow[t]{3}{*}{ Similarity } & \multicolumn{4}{|c|}{ Transcript level } \\
\hline & & & \multicolumn{2}{|c|}{ Low quality } & \multicolumn{2}{|c|}{ High quality } \\
\hline & & & Mean & SD & Mean & SD \\
\hline \multicolumn{7}{|c|}{ Down-regulated in lines with high dNDF content } \\
\hline \multicolumn{7}{|c|}{$>$ Chaperone activity } \\
\hline $\mathrm{A} 1948333$ & UP|TBB6_MAIZE Tubulin beta-6 chain & $100 \%$ & 0.13 & 0.06 & 0.10 & 0.16 \\
\hline \multicolumn{7}{|c|}{$>$ Catalytic activity } \\
\hline $\mathrm{Al} 666096$ & UP|SUS2_MAIZE Sucrose synthase 2 & $100 \%$ & 0.22 & 0.07 & 0.17 & 0.07 \\
\hline Al881378 & Zea mays clone Contig234 mRNA sequence & & 1.26 & 0.47 & 0.83 & 0.26 \\
\hline AW424679 & Zea mays clone ELOIN05I3EOI.d mRNA sequence & & 3.37 & 1.02 & 2.69 & 0.70 \\
\hline BG842237 & UP|Q6ZL43_ORYSA Histone H2A & $100 \%$ & 1.63 & 0.55 & 1.25 & 0.48 \\
\hline BM073983 & UP|Q9SXV0_ORYSA Cytochrome c oxidase subunit 6b-I & $44 \%$ & 2.96 & 1.06 & 1.92 & 0.68 \\
\hline CD97058I & PIR|T02767|T02767 cinnamyl-alcohol dehydrogenase - maize $\{$ Zea mays $\}$ & $100 \%$ & 2.12 & 0.72 & 1.31 & 0.65 \\
\hline CD973094 & UP|Q7MIQ5_ORYSA Phenylalanine ammonia-lyase & $96 \%$ & 1.91 & 0.68 & 1.73 & 0.60 \\
\hline$\overline{\text { CF028668 }}$ & UP|Q9SLP6_MAIZE Ferredoxin & $100 \%$ & 3.46 & 1.10 & 2.79 & 0.88 \\
\hline \multicolumn{7}{|l|}{$>_{\text {Binding }}$} \\
\hline Al734769 & UP|Q5DIM3_POPTR Class III HD-Zip protein 4 & $21 \%$ & 0.20 & 0.08 & 0.14 & 0.19 \\
\hline AW042324 & UP|Q9XEYI_TOBAC Nt-iaa2.3 deduced protein & $60 \%$ & 0.34 & 0.12 & 0.24 & 0.08 \\
\hline BG84I395 & UP|Q8RY5I_ORYSA Glucose-6-phosphate dehydrogenase & $100 \%$ & 1.96 & 0.60 & 1.45 & 0.58 \\
\hline BM080754 & UP|O24449_MAIZE Translational initiation factor elF-4A & $100 \%$ & 0.76 & 0.21 & 0.38 & 0.39 \\
\hline BM334752 & UP|Q7EYM0_ORYSA Zinc finger protein family-like & $98 \%$ & 3.52 & 1.05 & 2.91 & 0.93 \\
\hline \multicolumn{7}{|c|}{$>_{\text {Unclassified }}$} \\
\hline $\mathrm{Al} 622221$ & PRF|NP_191537 expressed protein $\{$ Arabidopsis thaliana\} & $42 \%$ & 1.00 & 0.33 & 0.68 & 0.20 \\
\hline $\mathrm{Al} 622354$ & UP|Q8GTX9_ARATH Cell cycle control crn (Crooked neck) protein-like & $17 \%$ & 0.81 & 0.33 & 0.43 & 0.17 \\
\hline $\mathrm{Al} 666222$ & UP|Q6CWV7_KLULA Kluyveromyces lactis strain NRRL Y-I I 40 & $5 \%$ & 1.73 & 0.62 & 1.12 & 0.34 \\
\hline $\mathrm{Al} 691363$ & GB|AFI 27565 ubiquitin-protein ligase 2 A Arabidopsis thaliana $\}$ & $3 \%$ & 0.37 & 0.14 & 0.23 & 0.08 \\
\hline $\mathrm{Al} 692057$ & UP|Q6K8U3_ORYSA Leucine-rich repeat-like protein & $29 \%$ & 0.80 & 0.30 & 0.59 & 0.21 \\
\hline Al737226 & UP|Q75GJ2_ORYSA Expressed protein (With alternative splicing) & $89 \%$ & 1.04 & 0.38 & 0.74 & 0.30 \\
\hline $\mathrm{Al} 834139$ & UP|Q6J9V5_MAIZE Cf2-like protein & $3 \%$ & 0.28 & 0.12 & 0.16 & 0.07 \\
\hline Al834361 & UP|C85AI_ORYSA Cytochrome P450 85AI (C6-oxidase) (Dwarf protein) & $10 \%$ & 1.09 & 0.38 & 0.68 & 0.26 \\
\hline Al855219 & & & 0.40 & 0.15 & 0.31 & 0.15 \\
\hline Al947947 & UP|Q6TY49_HYDMC Reductase I & $21 \%$ & 0.57 & 0.18 & 0.46 & 0.23 \\
\hline AW018225 & & & 1.86 & 0.75 & 1.55 & 0.61 \\
\hline AWI55860 & UP|Q5WAM8_BACSK DNA-directed RNA polymerase & $6 \%$ & 1.58 & 0.40 & 1.24 & 0.45 \\
\hline AW400279 & Zea mays clone ELOIN0325H04.d mRNA sequence & & 2.18 & 0.69 & 1.88 & 0.48 \\
\hline AW400378 & UP|Q9FU27_ORYSA CCCH-type zinc finger protein-like & $40 \%$ & 0.46 & 0.17 & 0.33 & 0.18 \\
\hline AW438085 & PRF|NP_56770I expressed protein $\{$ Arabidopsis thaliana\} & $55 \%$ & 0.98 & 0.47 & 0.41 & 0.27 \\
\hline AW787698 & UP|Q94FNI_LOTJA Phosphatidylinositol transfer-like protein III & $29 \%$ & 3.68 & 1.21 & 2.97 & 0.87 \\
\hline BG837856 & UP|Q9ZPI2_9BRAS Delta-12 desaturase & $46 \%$ & 2.41 & 0.73 & 1.64 & 0.54 \\
\hline BM333894 & UP|Q6SS00_ANTMA YABBY-like transcription factor GRAMINIFOLIA & $23 \%$ & 0.53 & 0.20 & 0.36 & 0.15 \\
\hline BM334482 & & & 2.89 & 0.83 & 2.17 & 0.67 \\
\hline BM349452 & PRF|NP_850290 expressed protein \{Arabidopsis thaliana\} & $28 \%$ & 0.78 & 0.55 & 0.09 & 0.08 \\
\hline BM350803 & UP|Q9PF60_XYLFA Endo-I, 4-beta-glucanase & $16 \%$ & 0.85 & 0.54 & 0.42 & 0.15 \\
\hline BM500212 & UP|Q9LQS9_ARATH T4OI2.9 & $39 \%$ & 0.61 & 0.33 & 0.18 & 0.14 \\
\hline CB350592 & & & 0.44 & 0.18 & 0.29 & 0.19 \\
\hline CF005238 & UP|Q9SBX4_MAIZE Proline-rich protein precursor & $51 \%$ & 3.06 & 1.09 & 2.11 & 0.38 \\
\hline CF062028 & UP|UPTG_MAIZE (P80607) Alpha-I, 4-glucan-protein synthase & $100 \%$ & 1.13 & 0.47 & 0.97 & 0.48 \\
\hline \multicolumn{7}{|c|}{ Up-regulated in lines with high dNDF content } \\
\hline \multicolumn{7}{|c|}{$>_{\text {Motor activity }}$} \\
\hline$\underline{A W 065585}$ & UP|Q9FUS4_SETIT Actin & $100 \%$ & 0.38 & 0.23 & 0.69 & 0.26 \\
\hline \multicolumn{7}{|c|}{$>_{\text {Catalytic activity }}$} \\
\hline Al622068 & UP|Q9LLI8_MAIZE Cellulose synthase-2 & $10 \%$ & 0.24 & 0.09 & 0.44 & 0.16 \\
\hline Al737885 & UP|O60585_HUMAN Ser/Arg-related nuclear matrix protein & $4 \%$ & 0.94 & 0.31 & 1.85 & 0.77 \\
\hline AW927815 & UP|TRXF_PEA (P29450) Thioredoxin F-type, chloroplast precursor & $59 \%$ & 0.24 & 0.11 & 0.48 & 0.20 \\
\hline BE050716 & Zea mays clone ELOIN055IB03.d mRNA sequence & & 0.08 & 0.03 & 0.12 & 0.06 \\
\hline BEI29940 & UP|PEPCK_MAIZE (Q9SLZ0) PEP carboxykinase & $100 \%$ & 0.42 & 0.15 & 0.92 & 0.37 \\
\hline BM334031 & UP|HMT2_MAIZE (Q9FUM9) Homocysteine S-methyltransferase 2 & $100 \%$ & 0.32 & 0.13 & 0.70 & 0.36 \\
\hline BM350652 & UP|Q45W77_ARAHY Ubiquitin-conjugating enzyme I & $100 \%$ & 0.68 & 0.28 & 1.40 & 0.60 \\
\hline
\end{tabular}


Table I: Differentially expressed ( $<$ 0.01) ESTs (102) between low and high digestible (dNDF) recombinant inbred lines. (Continued)

\begin{tabular}{|c|c|c|c|c|c|c|}
\hline CB617280 & Zea mays clone EL0IN053ID09.c mRNA sequence & & 0.14 & 0.05 & 0.29 & 0.16 \\
\hline$\underline{C D 972065}$ & UP|Q8L7Y9_ARATH Phosphatidylglycerol specific phospholipase C & $92 \%$ & 0.02 & 0.04 & 0.05 & 0.16 \\
\hline CF030921 & UP|Q9FNU9_DACGL Mitochondrial processing peptidase alpha-chain & $100 \%$ & 0.31 & 0.13 & 0.80 & 0.39 \\
\hline CF243853 & UP|Q7X6T0_MAIZE Caffeoyl CoA 3-O-methyltransferase & $100 \%$ & 0.03 & 0.06 & 0.06 & 0.20 \\
\hline \multicolumn{7}{|c|}{$>_{\text {Structural molecule activity }}$} \\
\hline$\underline{\mathrm{Al} 691512}$ & UP|TBA2_MAIZE Tubulin alpha-2 chain & $100 \%$ & 0.41 & 0.23 & 0.70 & 0.24 \\
\hline Al920382 & Zea mays clone Contig900.F mRNA sequence & & 0.38 & 0.14 & 0.66 & 0.23 \\
\hline \multicolumn{7}{|c|}{$>_{\text {Transporter activity }}$} \\
\hline Al621754 & UP|Q9LSD4_ARATH & $89 \%$ & 0.68 & 0.49 & 1.38 & 0.42 \\
\hline \multicolumn{7}{|l|}{$>_{\text {Binding }}$} \\
\hline AW40006I & UP|METK_ORYSA S-adenosylmethionine synthetase I & $100 \%$ & 0.16 & 0.06 & 0.43 & 0.19 \\
\hline AW438124 & UP|Q8W5I4_MAIZE MSI type nucleosome/chromatin assembly factor C & $100 \%$ & 0.18 & 0.07 & 0.35 & 0.14 \\
\hline BM078288 & PRF|NP_200425 KH domain-containing protein & $73 \%$ & 0.11 & 0.04 & 0.24 & 0.13 \\
\hline BM381996 & UP|O22470_ORYSA GDP dissociation inhibitor protein OsGDII & $80 \%$ & 0.07 & 0.03 & 0.15 & 0.08 \\
\hline \multicolumn{7}{|l|}{$>_{\text {Unclassified }}$} \\
\hline $\mathrm{Al} 622159$ & UP|O75833_HUMAN UTFI & $5 \%$ & 0.03 & 0.05 & 0.08 & 0.29 \\
\hline Al673886 & UP|O82347_ARATH Expressed protein (At2g46220/T3FI7.I3) & $61 \%$ & 0.02 & 0.03 & 0.04 & 0.15 \\
\hline$\underline{\mathrm{Al} 691788}$ & GB|BT0I0I34 At2g02560 \{Arabidopsis thaliana\} & $50 \%$ & 0.50 & 0.17 & 1.29 & 0.50 \\
\hline Al7|4870 & UP|Q7XVJ6_ORYSA OJ000I26_13.12 protein & $89 \%$ & 0.13 & 0.06 & 0.22 & 0.09 \\
\hline Al7|5073 & UP|Q4EVD5_LISMO ATP-dependent RNA helicase DeaD & $4 \%$ & 0.43 & 0.22 & 1.14 & 0.42 \\
\hline Al857233 & Zea mays clone EL0IN0442A09.c mRNA sequence & & 0.06 & 0.02 & 0.12 & 0.10 \\
\hline $\mathrm{Al} 1881373$ & UP|O24293_PEA Chloroplast inner envelope protein, II 0 kD precursor & $32 \%$ & 0.21 & 0.07 & 0.42 & 0.16 \\
\hline $\mathrm{Al} 1901469$ & UP|Q8GUG5_ARATH Threonine dehydratase/deaminase (OMRI) & $27 \%$ & 0.14 & 0.05 & 0.28 & 0.11 \\
\hline Al90I508 & UP|Q7TFG7_RHCM6 RhI75 & $11 \%$ & 0.09 & 0.07 & 0.27 & 0.38 \\
\hline Al920330 & UP|Q61683_ORYSA UDP-glucuronic acid decarboxylase & $91 \%$ & 0.59 & 0.22 & 0.89 & 0.36 \\
\hline $\mathrm{Al} 948303$ & & & 0.96 & 0.31 & 1.67 & 0.61 \\
\hline$\underline{\mathrm{Al} 964683}$ & UP|NRTN_HUMAN (Q99748) Neurturin precursor & $7 \%$ & 0.59 & 0.16 & 0.94 & 0.37 \\
\hline AW065798 & UP|RLI0_MAIZE 60S ribosomal protein LI0 & $39 \%$ & 0.26 & 0.07 & 0.38 & 0.13 \\
\hline AW091349 & UP|Q4NPG7_9DELT PE-PGRS family protein & $3 \%$ & 1.09 & 0.31 & 1.47 & 0.49 \\
\hline AWI20445 & UP|Q5UIM6_ORYSA Class III peroxidase 67 precursor & $53 \%$ & 2.06 & 0.73 & 3.42 & 1.15 \\
\hline AWI46725 & PRF|NP_19262I expressed protein $\{$ Arabidopsis thaliana\} & $60 \%$ & 0.31 & 0.09 & 0.48 & 0.17 \\
\hline AW330680 & UP|Q8L7U7_ARATH AT4g39670/TI9PI9_60 & $76 \%$ & 0.17 & 0.05 & 0.36 & 0.16 \\
\hline AW331016 & & & 0.36 & 0.12 & 0.78 & 0.33 \\
\hline AW331105 & PRF|NP_I89346 expressed protein $\{$ Arabidopsis thaliana\} & $77 \%$ & 0.19 & 0.08 & 0.47 & 0.32 \\
\hline AW331480 & UP|Q6ILL4_DROME HDC09080 & $8 \%$ & 0.15 & 0.07 & 0.29 & 0.12 \\
\hline AW400073 & UP|Q9FRX0_MAIZE Bundle sheath cell specific protein I & $100 \%$ & 0.14 & 0.04 & 0.27 & 0.12 \\
\hline AW438246 & PRF|NP_197590 expressed protein \{Arabidopsis thaliana\} & $20 \%$ & 0.26 & 0.13 & 0.45 & 0.20 \\
\hline$\underline{B E 05 I 493}$ & UP|Q9M0X9_ARATH 4-coumarate-CoA ligase-like protein & $34 \%$ & 0.29 & 0.12 & 0.70 & 0.32 \\
\hline BEI29888 & UP|Q6K702_ORYSA MATE efflux protein-like & $56 \%$ & 0.03 & 0.05 & 0.10 & 0.27 \\
\hline BF72878I & UP|Q9XEI4_MAIZE Unconventional myosin heavy chain & $10 \%$ & 0.31 & 0.12 & 0.70 & 0.31 \\
\hline BG321060 & PRF|NP_567470 expressed protein \{Arabidopsis thaliana\} & $23 \%$ & 0.08 & 0.05 & 0.16 & 0.09 \\
\hline$\underline{B G 842356}$ & UP|Q8L6II_ORYSA Plasma membrane H+ ATPase & $47 \%$ & 0.09 & 0.06 & 0.23 & 0.13 \\
\hline BG842835 & PRF|NP_195843 haloacid dehalogenase-like hydrolase family protein & $91 \%$ & 0.12 & 0.04 & 0.30 & 0.15 \\
\hline BM073617 & UP|Q56WY6_ARATH Serine protease like protein & $65 \%$ & 0.48 & 0.22 & 1.12 & 0.43 \\
\hline BM080336 & UP|Q9FZ9I_ARATH F3H9.17 protein (Sulfotransferase family protein) & $7 \%$ & 0.38 & 0.18 & 0.64 & 0.30 \\
\hline BM080577 & PRF|NP_193327 nodulin MtN3 family protein $\{$ Arabidopsis thaliana\} & $70 \%$ & 0.74 & 0.22 & 1.52 & 0.55 \\
\hline BM350621 & UP|Q4SGI8_TETNG Chromosome 3 SCAFI4593 & $5 \%$ & 0.29 & 0.09 & 0.78 & 0.36 \\
\hline BM382642 & UP|PSBQ I_MAIZE (Q4I048) Oxygen-evolving enhancer protein 3-I & $100 \%$ & 0.23 & 0.08 & 0.39 & 0.22 \\
\hline BQ279795 & Zea mays clone Contig8I5.F mRNA sequence & & 0.33 & 0.15 & 0.78 & 0.35 \\
\hline BU093700 & PRF|NP_680II 6 transcription factor jumonji family protein & $7 \%$ & 0.44 & 0.14 & 0.87 & 0.40 \\
\hline CB605303 & UP|Q56WY6_ARATH Serine protease like protein & $65 \%$ & 0.17 & 0.07 & 0.35 & 0.16 \\
\hline CB815513 & PRF|NP_850290 expressed protein \{Arabidopsis thaliana\} & $28 \%$ & 0.11 & 0.04 & 0.25 & 0.11 \\
\hline CD446744 & Zea mays clone EL0IT0206EOI.c mRNA sequence & & 0.15 & 0.06 & 0.27 & 0.12 \\
\hline CD972441 & UP|Q9XE75_SORBI Patatin-like protein & $100 \%$ & 0.24 & 0.10 & 0.42 & 0.19 \\
\hline CD984II2 & PRF|NP_I 74335 SIT4 phosphatase-associated family protein & $9 \%$ & 0.36 & 0.15 & 0.68 & 0.42 \\
\hline CD986361 & GB|AP00356I ankyrin-like protein \{Oryza sativa\} & $76 \%$ & 0.10 & 0.03 & 0.29 & 0.13 \\
\hline CD990067 & UP|C98AI_SORBI (O48956) Cytochrome P450 98AI & $100 \%$ & 0.21 & 0.07 & 0.43 & 0.17 \\
\hline CF031839 & Zea mays clone Contig483.F mRNA sequence & & 0.17 & $\begin{array}{c}0.0 \\
7\end{array}$ & 0.30 & $\begin{array}{c}0.1 \\
2\end{array}$ \\
\hline CF062473 & UP|Q8LBV7_ARATH plastid ribosomal protein LI9 & $33 \%$ & 0.15 & $\begin{array}{c}0.0 \\
7\end{array}$ & 0.29 & $\begin{array}{c}0.1 \\
7\end{array}$ \\
\hline
\end{tabular}

a GenBank accession number.

b Annotation of each gene sequence was taken from the TIGR Maize Gene Index [12]. 
Table 2: 20 flint inbred lines each, out of a population of $\mathbf{2 7 0}$ recombinant inbred lines with the lowest or highest dNDF values, respectively.

\begin{tabular}{|c|c|c|c|}
\hline Low quality lines & dNDF $^{\mathrm{a}}(\%)$ & High quality lines & dNDF (\%) \\
\hline LOI & 45.21 & $\mathrm{HOI}$ & 60.87 \\
\hline L02 & 50.07 & $\mathrm{H} 02$ & 60.97 \\
\hline L03 & 50.41 & $\mathrm{H} 03$ & 61.03 \\
\hline L04 & 50.72 & $\mathrm{H} 04$ & 61.11 \\
\hline L05 & 51.37 & $\mathrm{H} 05$ & 61.15 \\
\hline L06 & 51.37 & $\mathrm{H} 06$ & 61.24 \\
\hline L07 & 51.80 & $\mathrm{H} 07$ & 61.28 \\
\hline L08 & 52.55 & $\mathrm{H} 08$ & 61.42 \\
\hline L09 & 52.78 & $\mathrm{H} 09$ & 61.85 \\
\hline LIO & 52.78 & $\mathrm{HIO}$ & 61.86 \\
\hline LII & 53.46 & $\mathrm{HII}$ & 61.99 \\
\hline $\mathrm{L} 12$ & 53.47 & $\mathrm{HI} 2$ & 62.10 \\
\hline LI3 & 53.72 & $\mathrm{HI3}$ & 62.44 \\
\hline LI4 & 53.75 & $\mathrm{HI} 4$ & 62.78 \\
\hline LI5 & 53.77 & $\mathrm{HI} 5$ & 62.87 \\
\hline LI6 & 53.82 & $\mathrm{HI} 6$ & 63.38 \\
\hline $\mathrm{LI7}$ & 53.86 & $\mathrm{HI7}$ & 63.52 \\
\hline LI8 & 53.94 & $\mathrm{HI} 8$ & 63.99 \\
\hline LI9 & 53.94 & $\mathrm{HI9}$ & 64.96 \\
\hline L20 & 53.95 & $\mathrm{H} 20$ & 65.22 \\
\hline Mean & 52.34 & Mean & 62.30 \\
\hline SD & 2.12 & SD & 1.32 \\
\hline
\end{tabular}

a the percentage of digestible neutral detergent fiber.

Maize genetic maps have been divided into 100 segments (=bins) of approximately $20 \mathrm{cM}$ length [3]. In total, 271 eQTL were detected in 24 bins covering all chromosomes except for chromosome 7 (Figure 5b). The percentage of eQTL per bin was plotted against chromosomal bins (Figure 5). eQTL hotspots, containing more than $5 \%$ of the total number of eQTL, were detected on bins $1.07,1.12$, $3.05,8.03$, and 9.04. Out of those, bin 3.05 co-localized with one of five major QTL clusters for cell-wall digestibility (Figure 6), which were identified on bins 1.03, 3.05/ $06,6.06,8.05$, and $9.02[1]$.

Out of 89 ESTs resulting in eQTL, for 14 ESTs bin information from the Maize GDB [13] and map positions from Maize Genome Mapping project [14] (Figure 6) are known. For another 10 ESTs, only chromosomal but not bin assignment were provided from Maize Genome Mapping project. In silico mapping information indicate that the ESTs were located in various linkage groups, and generally not in the genetic location of the respective eQTL. AW438124 (nucleosome/chromatin assembly factor C), BM080577 (nodulin MtN3 family protein), and AI881378 (maize clone contig234 mRNA sequence) were co-localized with a cell-wall digestibility related QTL cluster on bin 3.05 .

\section{Discussion}

\section{Power of eQTL analysis}

An essential first step in QTL analysis is to assess how many samples must be collected in order to achieve sufficient power to detect a hypothetical effect. eQTL mapping is different from QTL mapping of complex inherited traits, since we expect (and are mainly interested in) major eQTL due to 1) eQTL in cis (then most of the variation should be explained by this one eQTL), and 2) major regulatory eQTL (one or few) in trans. Thus, we expect a simpler inheritance of expression patterns of single ESTs with only one or few eQTL involved, each explaining a comparatively large percentage of the phenotypic variation, in contrast to "true" quantitative characters such as grain yield. Major eQTL should be detectable even with this very limited number of RILs. In addition, this requirement is particularly salient in eQTL analysis due to the high expenses of gene-expression analyses. In plants, both $76 \mathrm{~F} 3$ families in maize [10] and $91 \mathrm{BC} 1$ families in poplar [11] were used in respective eQTL analysis. In this study, 40 RIL lines were used to detect eQTL in the FF population. Because of an increased heritability when using homozygous and homogeneous RIL lines as compared to segregating F2:3 families, an eQTL explaining $28 \%$ of the variation in RNA abundance among the F2 population 
a)

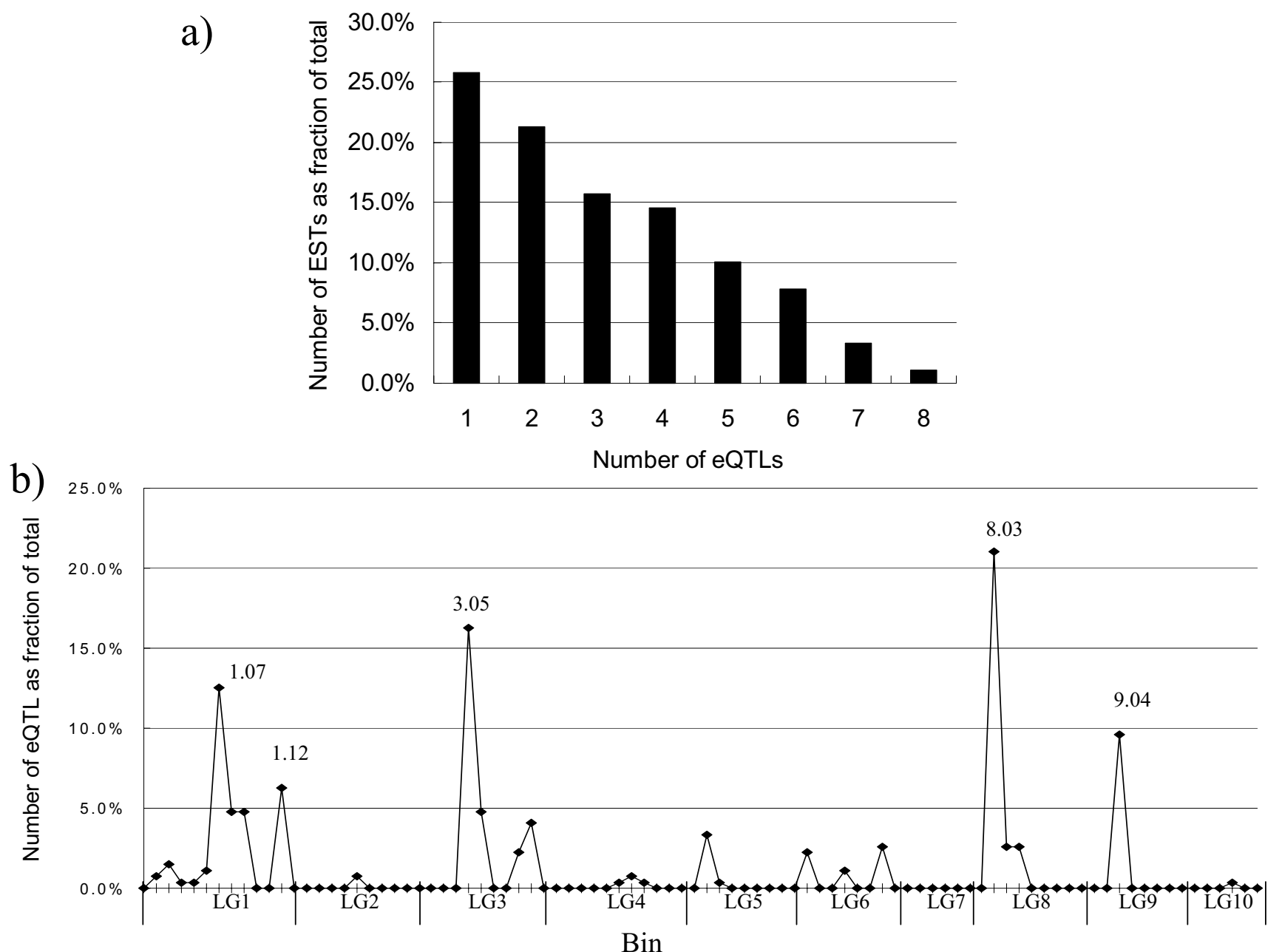

b)

Figure 5

Expression quantitative trait loci (eQTL) (LOD $\geq 2.4$ ) distributions. a) Distribution of numbers of eQTLs detected for corresponding EST; b) Genome-wide distribution of eQTLs according to the bins.

would be detected in $99 \%$ of the experiments with 40 RI lines, in contrast to $94 \%$ of the experiments performed with 76 F3 families [9]. In addition, at least 73\% of the experiments with 40 RIL lines should detect an eQTL explaining $18 \%$ of the phenotypic variance [9]. Since $79 \%$ (214 of 271) identified eQTL explained more than $28 \%$ of the transcription variation of the corresponding ESTs and minimum eQTL explaining $24.1 \%$ transcription variation, 40 extreme lines should be sufficient to detect major eQTL. However, the explained phenotypic variance by individual $\mathrm{eQTL}$ is very likely overestimated due to the small population size [15].

\section{Genetic architecture of transcription}

Genetical genomics permits quantitative assessment of the proportion of gene loci (ESTs) displaying co-segregation with the respective eQTL (cis-eQTL), relative to those that produce unlinked trans-eQTL [13]. In maize ear leaf, $34 \%(6481$ of 18,805$)$ differentially expressed genes produced cis-eQTL with LOD $>3.0$ [10]. In contrast, no in silico mapped ESTs were in the same location as their own eQTL in this study (Figure 6). Accordingly, no key genes involved in lignin biosynthesis co-localized with cis-eQTL in poplar [11]. Therefore, trans-acting factors could play a major role in regulating heritable variation of gene expression in cell wall digestibility.

Only 26\% (23 of 89) ESTs detected a single eQTL in the present study. For the remaining $74 \%$, two or more eQTL were found (Figure 5). A similar distribution of eQTL was found in yeast [16]. Only 3\% transcripts were consistent with single locus inheritance, $18 \%$ suggested control by two loci, and $>50 \%$ required at least five loci under an additive model. Thus, transcriptional variation was gener- 


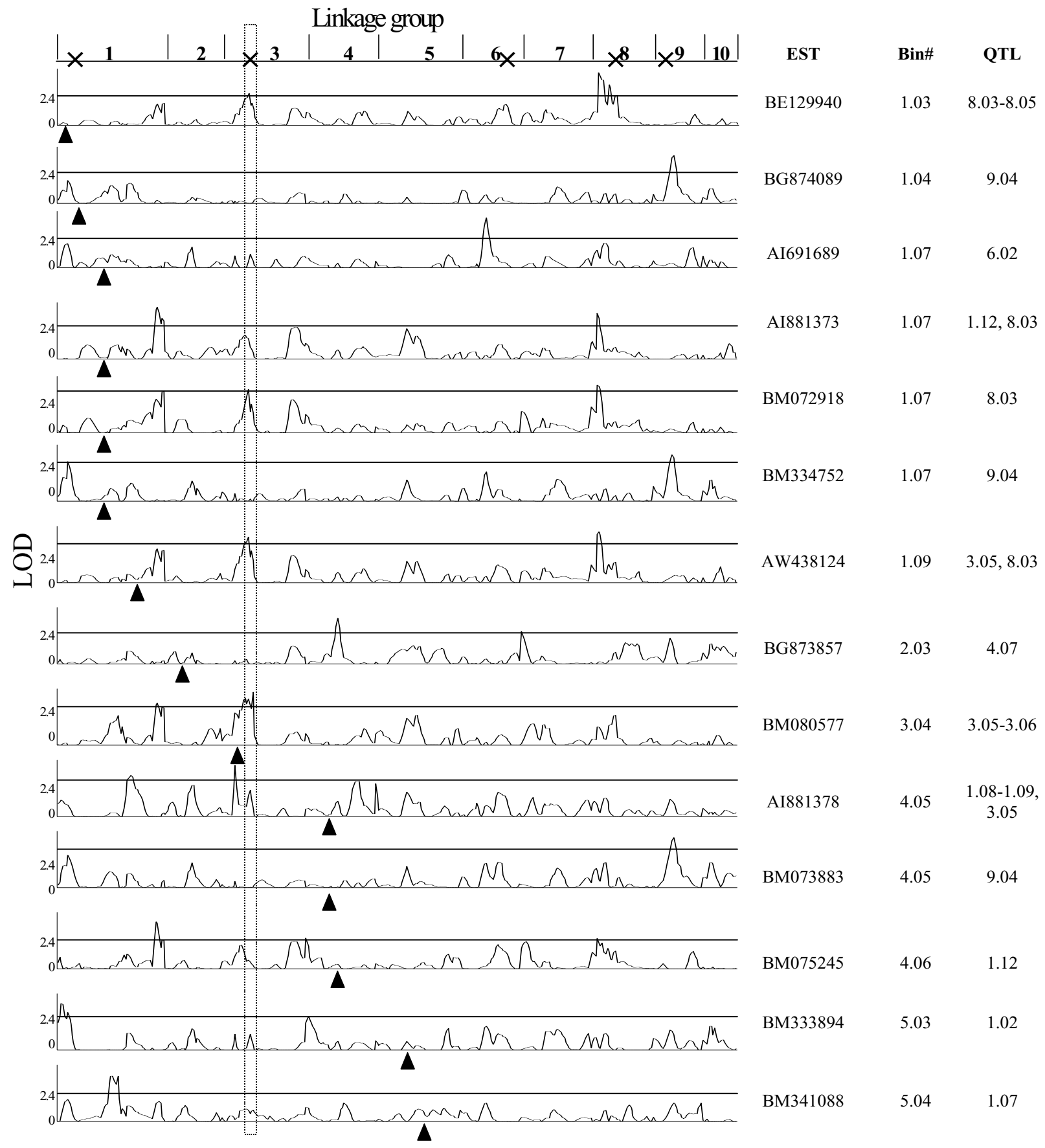

\section{Figure 6}

LOD score profiles generated by interval mapping analysis of dNDF and expression levels of I4 ESTs in silico mapped among 89 ESTs resulting in eQTL. The black triangles indicate the in silico mapping position for each EST. EST mapping information was extracted from the Maize GDB [13] and the IDP mapping project [27], according to map bins [3]. The $x$ axis represents ten linkage groups of the Flint $\times$ Flint genetic map arranged end-to-end. The middle line represents LOD score threshold 2.4. The " $x$ " indicates five major QTL clusters for cell wall digestibility on bins I.03, 3.05/06, 6.06, 8.05, and 9.02 [I]. The dashed rectangle shows some eQTLs co-localizing with one major QTL cluster for cell wall digestibility on bin 3.05 . 
ally found to be oligogenic rather than monogenic inherited.

A common feature of eQTL studies is the detection of "hotspots" or hubs of trans-acting eQTL: chromosomal regions that affect the expression of a much larger number of genes than expected by chance [9]. eQTL hotspots, containing more than $5 \%$ of the total number of eQTL, were found on bins 1.07, 1.12, 3.05, 8.03, and 9.04 (Figure 5). The strong clustering in hubs of eQTL reflects highly correlated expression levels of many gene transcripts in association with cell-wall digestibility. The five hotspots could contain important transcript factors for cell wall digestibility. However, no obvious transcription factors were found among those genes currently mapped to these bin regions [13]. Similarly, eQTL identified in yeast frequently resulted from genetic variants that altered activity of a signaling or metabolic pathway without directly altering gene transcription [17]. 4CL2, F5H, and CCR1 genes involved in lignin biosynthesis map to bin 1.07 [1]. These genes are candidates for the eQTL found in the same genome region due to potential metabolic feedback mechanisms acting through the same enzyme(s) in lignin biosynthesis. However, due to the limited understanding of genetic and physiological control of gene expression so far, any conclusions with regard to hotspots for gene regulation should be interpreted with caution [9]. Kirst et al. [11] found that two eQTL hubs for lignin-related genes co-localized with growth QTL on linkage groups 4 and 9 in poplar. One eQTL hotspot was co-localized with cell wall digestibility related QTL cluster [1] on bins 3.05 (Figure 6), implying that in this case the gene(s) underlying QTL and eQTL are identical.

\section{Molecular mechanisms underlying cell-wall digestibility} Lignin restricts the degradation of structural polysaccharides by hydrolytic enzymes, thereby limiting the bioconversion of forages into animal products [18]. Out of 102 significantly differentially expressed ESTs between high and low quality groups (Table 2), four ESTs encode enzymes involved in lignin biosynthesis, CD970581 (Cinnamyl-alcohol dehydrogenase), CD973094 (Phenylalanine ammonia-lyase), BE051493 (4-coumarate-CoA ligase-like protein), and CF243853 (Caffeoyl CoA 3-Omethyltransferase). In agreement with the expectation of low levels of lignin, CD970581 and CD973094 were down-regulated in lines with high forage quality. However, BE051493 and CF243853 were up-regulated, which might be due to the complexity of cell wall biosynthesis. The associations between one lignin characteristic (e.g., lignin composition) and cell wall degradability can be confounded and correlated to concurrent changes in other lignin properties (e.g., lignin cross-linking) that influence cell wall degradability [18]. Since reduced cellulose synthesis invoked lignification and defense responses in Ara- bidopsis [19], repressed ESTs encoding cellulose synthesis enzymes, such as AI622068 (Cellulose synthase-2) (Table 2 ), could activate lignin synthesis in low quality lines. In addition, four regulatory genes, AI734769 (Class III HDZip protein 4), BM080754(Translational initiation factor eIF-4A), BM334752 (Zinc finger protein family-like), and BM333894 (YABBY-like transcription factor), were downregulated in lines with high forage quality, whereas one regulatory gene, BU093700 (transcription factor and jumonji family protein) was up-regulated (Table 2 ). These regulatory genes might control cell wall digestibility spatially and temporally among the two groups of lines in the FF population. Mapping of these genes will reveal, whether they colocalize with eQTL (clusters) identified in this study. Furthermore, there are a number of genes, where a connection to digestibility is unclear, some of which might be false positives.

Using cellular UV-microspectrophotometry, Shi et al. [5] demonstrated that lignin content represented by the absorbance values within the cells of sclerenchyma fiber tissue was decreasing from the periphery to the center. Lignin content was lowest in S2 (secondary cell wall) of parenchyma cells, followed by S2, CML (compound middle lamella) and CC (cell corner) of sclerenchyma fibers. Each cell type in the stem likely expresses a unique transcriptome. Transcriptome analysis using complete stems provides average gene expression levels integrated over all cell types. In order to further understand the molecular basis of cell wall degradability, new methods, for instance, laser-capture microdissection [20], are needed for efficient isolation of CC of sclerenchyma fiber from different time points to further validate 439 candidate genes.

\section{Application of genetical genomics to plant breeding}

The use of functional genomics is contributing to many aspects of QTL analysis and cloning [21]. To date, most plant QTL have been cloned using a positional cloning approach following identification in experimental crosses. Transcriptional profiling can quickly provide a list of differentially expressed genes between contrasting QTL genotypes. Subsequently, those genes functionally related to the target trait and mapping to the QTL region can be selected as candidates. Unfortunately, the number of QTL cloned to date in plants is too small to test the validity of this approach. If the difference in gene expression level between alleles is too low (approximately twofold), the candidate genes cannot be identified using standard microarray-based transcriptome analysis [21]. Less than $15 \%$ of ESTs were induced more than two-fold by microarray analyses in FD-pop $(12 \%, 1,417$ of 11,827$)$ (Figure $1)$, DD1-pop (3\%, 317 of 11,827) and DD2-pop (15\%, 1,805 of 11,827), respectively. A genetical genomics approach might be better suited for detecting differences in gene expression. 23\% of the ESTs on the Forage Quality 
Array (102 of 439) were significantly different between low and high digestible (dNDF) RILs. However, this higher proportion of significantly differentially expressed genes identified by the genetical genomics approach is most likely due to successful selection of candidates by the criteria outlined above (Figure 2).

Since most of the important agronomic traits are complex inherited traits, transcript abundance may act as an intermediate phenotype between genomic DNA sequence variation and complex traits. Integration of genome-wide expression profiling with linkage analysis is a new approach to identifying genes underlying complex traits. Hubner et al. [22] has demonstrated large-scale identification of positional candidates and regulatory pathways for previously mapped physiological QTL by genetical genomics approach in the rat. Cis-acting eQTL are good candidates for physiological QTL because they show linespecific differences in gene expression that are under the control of DNA sequence variants in or close to the gene itself [23]. Similarly, eQTL identified in an intercross of inbred C57BL/6J and DBA/2J mice could accurately identify transcripts in which expression was regulated by cisacting polymorphisms [24]. Cis-acting eQTL might be detected by additional EST mapping or indirectly through the ongoing maize genome sequencing project, since so far only 24 of 89 ESTs resulting in eQTL were in silico mapped. Trans-acting eQTL represent loci that influence expression of genes or transcripts remote from the eQTL itself. Coincidental mapping of trans-acting eQTLs for multiple transcripts to the same chromosomal location, as observed on bin 3.05 (Figure 6), may represent a shared regulatory transcriptional control mechanism by a single gene at the eQTL. The locations of trans-acting eQTL in relation to physiological QTL, together with the locations of cis-acting eQTL, may point to genes and regulatory pathways underlying individual cell wall digestibility related QTL.

However, the scope of genetic analysis of gene expression also presents enormous technical and analytical challenges. Extraordinarily large number of comparisons were involved in a genome-wide linkage scan for several hundreds or thousands of transcripts, thus technical and experimental design issues need to be addressed to handle the large data sets that are being generated, and new statistical tools are still being evaluated [25]. As the field of genetical genomics develops, it is expected to significantly improve our knowledge about complex traits, such as cell wall degradability [8]. Therefore, in the future, it is conceivable that QTL cloning will increasingly rely on candidate gene information. Comprehensive knowledge of the lignin pathway and cell wall biogenesis will allow plant breeders to choose the best genomic targets controlling these characters, for improving forage digestibility through genetic engineering or marker-assisted selection.

\section{Conclusion}

102 candidate genes for cell-wall digestibility were validated by genetical genomics approach. Although the cDNA array highlights gene types (the tested gene and any close family members), trans-acting factors or metabolic bottlenecks seem to play the major role in controlling heritable variation of gene expression related to cell-wall digestibility, since no in silico mapped ESTs were in the same location as their own eQTL. Transcriptional variation was generally found to be oligogenic rather than monogenic inherited due to only $26 \%$ ESTs detected a single eQTL in the present study. One eQTL hotspot was colocalized with cell wall digestibility related QTL cluster on bins 3.05, implying that in this case the gene(s) underlying QTL and eQTL are identical. As the field of genetical genomics develops, it is expected to significantly improve our knowledge about complex traits, such as cell wall degradability. Comprehensive knowledge of the lignin pathway and cell wall biogenesis will allow plant breeders to choose the best genomic targets controlling these characters, for improving forage digestibility through genetic engineering or marker-assisted selection.

\section{Methods \\ Plant materials}

AS20, AS21, recombinant inbred lines (RILs), and doubled haploid (DH) lines were obtained from KWS Saat $A G$, including twenty highly digestible and twenty low digestible lines each in four mapping populations: FD (Flint $\times$ Dent DH population, AS08 $\times$ AS 06), DD1 (Dent $\times$ Dent DH population, AS11 $\times$ AS09), DD2 (Dent $\times$ Dent DH population, AS29 $\times$ AS30), and FF (Flint $\times$ Flint RIL population, AS18 $\times$ AS07) RIL mapping populations, respectively. These lines can be obtained from KWS Saat AG for non-commercial research purposes. All plants were grown and maintained in the greenhouse under a $12 \mathrm{~h}$ photoperiod at $23^{\circ} \mathrm{C}$ and $50 \%$ relative humidity. Stems were harvested 5 weeks after germination, since the highest level of COMT expression was observed in stems 5 and 7 weeks after germination [5]. For biological replication, two independent sets of three plants per set were harvested for AS20, AS21, and every line in FD, DD1, and DD2 populations, respectively, and one set of ten plants per line in the FF population. Since the maize stalk is small and covered by leaf sheath at early vegetative stages, the stem fraction investigated in this study is a mixture of stalk and leaf sheath [26]. Total RNA was extracted from the stems of each set of each line using TRIzol reagent (Invitrogen, Carlsbad, CA, USA). 


\section{Construction and hybridisation of Forage Quality Array} The macroarrays containing SSH clones were derived from the studies on three sets of maize brown-midrib isogenic lines in the genetic background of inbreds 1332 (1332 and $1332 \mathrm{bm} 3$ ), 5361 (5361 and $5361 \mathrm{bm} 3$ ), and F2 (F2, $\mathrm{F} 2 \mathrm{bm} 1, \mathrm{~F} 2 \mathrm{bm} 2$, and F2 bm3) [5]. In total, 2688 clones were spotted in duplicate on each macroarray. These clones were randomly picked from five SSH libraries. For two stem pairs (5361 vs. 5361 bm3; 1332 vs. 1332 bm3) subtractions were conducted in both directions. For the root pair 1332 versus 1332 bm3 the hybridization was performed only in forward direction. Microarray hybridization data were evaluated by the SpotReport ${ }^{\mathrm{TM}}$ Alien $^{\mathrm{TM}} \mathrm{cDNA}$ Array Validation System (Stratagene, La Jolla, CA, USA), including positive, negative, and 10 spiking controls. Out of 2688 clones, 1,401 clones, ranging in length from 74 to 989 bp, were sequenced by MWG (Ebersberg, Munich, Germany) and clustered into 765 ESTs. Their sequences were analyzed in the same way as microarray-ESTs.

In the FD, DD1, and DD2 populations, the total RNA representing high and low forage quality $\mathrm{DH}$ lines was pooled from the total RNA of those twenty lines in each of the populations with the highest or lowest digestibility (DNDF), respectively. The pooled total RNA and the total RNA of AS20 and AS21 were labeled with ${ }^{32} \mathrm{P}$ and hybridized to different arrays using the Strip-EZ RT kit (Ambion, Austin, TX, USA). Hybridization signals were detected by the Storm 860 imaging system (Amersham) with a resolution of $50 \mu \mathrm{m}$. After scanning, labeled cDNA probes were completely stripped from the arrays using the Strip-EZ system (Ambion). Each array was hybridized four times with four replicates per line, including two biological replications and two labeling replications in each biological replica.

Maize unigene-microarrays were provided by the laboratory of Prof. Schnable (Iowa University, USA) and contained 11,827 maize ESTs [27]. Poly (A)+ RNA was isolated from the same total RNA for probe preparation in the SSH approach via Dynabeads ${ }^{\circledast}$ Oligo(dT)25 (Dynal biotech, Oslo, Norway). According to TIGR Microarray Protocols [28], each mRNA sample was indirectly labeled with Cy3 or Cy5 (Amersham Pharmacia, Piscataway, NJ, USA) and hybridized with maize unigene-microarrays. Fluorescence signals were detected using the arrayWoRx ${ }^{\circledR}$ Biochip Reader (Applied Precision, Issaquah, WA, USA). For high vs. low digestibility lines in FD, DD1, and DD2 populations as well as AS20 vs. AS21, four replications including two biological and two dye-swap replications within each biological replication were conducted. Thus, four maize gene chips were used in each comparison.

The raw images obtained from Storm 860 imaging system or arrayWoRx ${ }^{\circledR}$ Biochip Reader were imported into Array-
Vision 8.0 (Imaging Research, St. Catharines, Ontario, Canada) for spot detection and quantification of hybridization signals. Raw data exported from ArrayVision 8.0 were imported into Excel and converted to TIGR Array Viewer (TAV) format files. Data were normalized using intensity-dependent local regression (Lowess) implemented in the Microarray Data Analysis System (MIDAS) [29]. All calculated gene expression ratios were log 2transformed and averaged over four replicates in each comparison. Differentially expressed ESTs at the 95\% confidence level were determined using intensity-dependent Z-scores (with $Z=1.96$ ) as implemented in MIDAS and the union of all genes identified in each comparison was considered significant in this experiment. Each EST was annotated and assigned with GO vocabularies according to the TIGR Maize Gene Index [12]. The mapped ESTs contain bin information from the Maize GDB [13] and the IDP mapping project [27].

Among 865 candidate ESTs for cell wall digestibility, 208 ESTs were ordered from the Iowa Schnable Lab [30] and 506 ESTs from the Arizona BAC/EST resource center [31]. From the stab cultures of these ESTs, plasmid minipreps were conducted by use of the R.E.A.L. Prep 96 Plasmid Kit (Qiagene AG, Germany). Using the plasmids of each EST as template, two independent re-amplification $(100 \mu \mathrm{l})$ reactions were performed and pooled to reduce the effects of variation in PCR efficiency. Pooled PCR products were concentrated from $200 \mu \mathrm{l}$ to approximately $25 \mu \mathrm{l}$ using MultiScreen-PCR plates (Millipore, Billerica, MA, USA). Due to poor bacteria recovery and unspecific PCR amplification, high quality PCR products were derived from only 439 ESTs and spotted on Nexterion ${ }^{\circledR}$ Slide A+ glass slides (SCHOTT Jenaer Glas GmbH, Germany) in a triple pattern using a QarrayMini spotter (Genetix GmbH, Germany). The total RNA of each line in the FF population was labeled and hybridized in the same way as the total RNA of pooled lines in the other three populations. Using loop design [32], each Forage Quality Array was hybridized with two contrasting lines (Table 1) labeled with different dyes. In total, 40 Forage Quality Arrays were used and each line was employed with dye-swap replication.

\section{Data analysis of the "genetical genomics" experiment}

The raw images of Forage Quality Arrays obtained from a GeneTAC $^{\mathrm{TM}}$ UC4 Microarray Scanner (Genomics Solutions Ltd., USA) were imported into ArrayVision 8.0 for spot detection and quantification of hybridization signals. In ArrayVision 8.0, Lowess normalization was implemented to remove dye effects. The transcript level of each EST was represented by the average over six measures, including dye swap replication and three in-slide replications within either Cy3 or Cy5 replications. 
The dataset of all 40 lines in the FF population were imported in Multiexperiment Viewer (MeV) [29]. "Hierarchical cluster analysis" [33] was conducted to discover similar expression patterns across 439 ESTs, and "between subject t-tests $(p<0.05)$ " [34] were utilized to identify candidate genes differentially expressed between high and low digestible lines.

A complete linkage map was constructed by KWS Saat AG with 156 loci using 270 lines, including forty extreme lines, in FF population. Low quality lines have all unfavorable QTL/alleles for $\mathrm{dNDF}$, and high quality all favorable QTL/alleles for dNDF. The same mapping population was used for both QTL (Krützfeldt et al., in preparation) and eQTL mapping. Five dNDF QTLs were identified, and the positions of the QTL were in good agreement with previous QTL studies [1]. Identification of QTL for gene expression traits was performed based on the 40 extreme lines using interval mapping implemented in MapQTL [35]. An empirical LOD score threshold of 2.4 was determined by permutation testing $[36,37]$ and adopted from Kirst et al. [11].

Original microarray expression data presented in this manuscript are available through ArrayExpress [38] with accession number E-MEXP-253.

\section{Authors' contributions}

CS carried out the most of experiments, performed the data analysis, and drafted the manuscript. AU carried out the hybridization of Forage Quality Arrays. MO conceived of the study and participated in its coordination. ML participated in the design of the study. GW conceived of the study and assisted CS with the experiments. TL conceived of the study, participated in its design, and drafted the manuscript. All authors read and approved the final manuscript.

\section{Acknowledgements}

We thank Preben B. Holm, Giuseppe Dionisio, and Per Gregersen for assistance with microarray experiments in Research Centre Flakkebjerg. We also thank Birte Krützfeldt and Joachim Eder for measuring dNDF values of extreme lines. This work was supported by EUREKA project $\Sigma ! 2386$ CEREQUAL.

\section{References}

I. Ralph J, Guillaumie S, Grabber JH, Lapierre C, Barriere Y: Genetic and molecular basis of grass cell-wall biosynthesis and degradability. III. Towards a forage grass ideotype. Comptes Rendus Biologies 2004, 327(5):467-479.

2. Barriere $Y$, Guillet $C$, Goffner D, Pichon M: Genetic variation and breeding strategies for improved cell wall digestibility in annual forage crops. A review. Animal Research 2003, 52(3): 193-228.

3. Gardiner JM, Coe EH, Melia-Hancock S, Hoisington DA, Chao S: Development of a core RFLP map in maize using an immortalized F2 population. Genetics 1993, 134(3):917-930.

4. Barriere Y, Argillier O: Brown-Midrib Genes of Maize - a Review. Agronomie 1993, I3(10):865-876.
5. Shi C, Koch G, Ouzunova M, Wenzel G, Zein I, Lubberstedt T: Comparison of maize brown-midrib isogenic lines by cellular $U V$ microspectrophotometry and comparative transcript profiling. Plant Molecular Biology 2006, 62(4-5):697-7|4.

6. Borevitz JO, Chory J: Genomics tools for QTL analysis and gene discovery. Curr Opin Plant Biol 2004, 7(2): I32-136.

7. Jansen RC, Nap JP: Genetical genomics: the added value from segregation. Trends Genet 200I, I 7(7):388-39I.

8. Li J, Burmeister M: Genetical genomics: combining genetics with gene expression analysis. Human Molecular Genetics 2005, I4:RI63-RI69.

9. de Koning DJ, Haley CS: Genetical genomics in humans and model organisms. Trends Genet 2005, 2 I (7):377-38I.

10. Schadt EE, Monks SA, Drake TA, Lusis AJ, Che N, Colinayo V, Ruff TG, Milligan SB, Lamb JR, Cavet G, et al.: Genetics of gene expression surveyed in maize, mouse and man. Nature 2003, 422(6929):297-302.

II. Kirst M, Myburg AA, De Leon JPG, Kirst ME, Scott J, Sederoff R: Coordinated genetic regulation of growth and lignin revealed by quantitative trait locus analysis of cDNA microarray data in an interspecific backcross of eucalyptus. Plant Physiology 2004, I35(4):2368-2378.

12. TIGR Maize Gene Index [http://compbio.dfci.harvard.edu/tgi/ plant.html]

13. The Maize GDB [http://www.maizegdb.org]

14. Maize Genome Mapping project [http://maize-mapping.plantge nomics.iastate.edu/]

15. Melchinger AE, Utz HF, Schon CC: Quantitative trait locus (QTL) mapping using different testers and independent population samples in maize reveals low power of QTL detection and large bias in estimates of QTL effects. Genetics 1998, I49(I):383-403.

16. Brem RB, Kruglyak L: The landscape of genetic complexity across 5,700 gene expression traits in yeast. Proceedings of the National Academy of Sciences of the United States of America 2005, 102(5): 1572-1577.

17. Brem RB, Yvert G, Clinton R, Kruglyak L: Genetic dissection of transcriptional regulation in budding yeast. Science 2002, 296(5568):752-755.

18. Grabber JH: How do lignin composition, structure, and crosslinking affect degradability? A review of cell wall model studies. Crop Science 2005, 45(3):820-83I.

19. Cano-Delgado A, Penfield S, Smith C, Catley M, Bevan M: Reduced cellulose synthesis invokes lignification and defense responses in Arabidopsis thaliana. Plant Journal 2003, 34(3):35I-362.

20. Schnable PS, Hochholdinger F, Nakazono M: Global expression profiling applied to plant development. Curr Opin Plant Biol 2004, 7(I):50-56.

21. Salvi S, Tuberosa R: To clone or not to clone plant QTLs: present and future challenges. Trends in Plant Science 2005, I0(6):297-304.

22. Hubner N, Wallace CA, Zimdahl H, Petretto E, Schulz H, Maciver F, Mueller M, Hummel O, Monti J, Zidek V, et al.: Integrated transcriptional profiling and linkage analysis for identification of genes underlying disease. Nat Genet 2005, 37(3):243-253.

23. Sladek R, Hudson TJ: Elucidating cis- and trans-regulatory variation using genetical genomics. Trends Genet 2006, 22(5):245-250.

24. Doss S, Schadt EE, Drake TA, Lusis AJ: Cis-acting expression quantitative trait loci in mice. Genome Res 2005, I5(5):68I-69I.

25. Gibson G, Weir B: The quantitative genetics of transcription. Trends Genet 2005, 2 I (I I ):6 16-623.

26. Corn production [http://maize.agron.iastate.edu/corn
a grows.html\#stages]

27. Maize chip [http://www.plantgenomics.iastate.edu/maizechip/]

28. Hegde P, Qi R, Abernathy K, Gay C, Dharap S, Gaspard R, Hughes JE, Snesrud E, Lee N, Quackenbush J: A concise guide to cDNA microarray analysis. Biotechniques 2000, 29(3):548-550. 552-544, 556 passim

29. Saeed Al, Sharov V, White J, Li J, Liang W, Bhagabati N, Braisted J, Klapa M, Currier T, Thiagarajan M, et al.: TM4: a free, open-source system for microarray data management and analysis. Biotechniques 2003, 34(2):374-378.

30. The lowa Schnable Lab [http://schnablelab.plantgenom ics.iastate.edu/research/genomics/htp est/ordering.php] 
31. The Arizona BAC/EST resource center [http://genome.ari zona.edu/orders/]

32. Churchill GA: Fundamentals of experimental design for cDNA microarrays. Nature Genetics 2002, 32:490-495.

33. Eisen MB, Spellman PT, Brown PO, Botstein D: Cluster analysis and display of genome-wide expression patterns. Proc Natl Acad Sci U S A 1998, 95(25): | 4863-14868.

34. Pan W: A comparative review of statistical methods for discovering differentially expressed genes in replicated microarray experiments. Bioinformatics 2002, I8(4):546-554.

35. Ooijen V, W. J, Boer MP, Jansen RC, Maliepaard C: MapQTL 4.0, Software for the caculation of QTL position on genetic maps. Wageningen, the Netheerlands: Plant Research International; 2002.

36. Churchill GA, Doerge RW: Empirical Threshold Values for Quantitative Trait Mapping. Genetics 1994, I38(3):963-97I.

37. Doerge RW, Churchill GA: Permutation tests for multiple loci affecting a quantitative character. Genetics 1996, I 42(1):285-294.

38. ArrayExpress [http://www.ebi.ac.uk/arrayexpress]

Publish with Bio Med Central and every scientist can read your work free of charge

"BioMed Central will be the most significant development for disseminating the results of biomedical research in our lifetime. "

Sir Paul Nurse, Cancer Research UK

Your research papers will be:

- available free of charge to the entire biomedical community

- peer reviewed and published immediately upon acceptance

- cited in PubMed and archived on PubMed Central

- yours - you keep the copyright

Submit your manuscript here:

http://www.biomedcentral.com/info/publishing_adv.asp
BiolMedcentral 